\title{
Combination of interval-valued neutrosophic soft sets and graph theory
}

\author{
Yildiray Celik and Guven Kara \\ Ordu University, Department of Mathematics, Ordu, Turkey
}

Received: 15 August 2017, Accepted: 17 September 2017

Published online: 7 April 2018.

\begin{abstract}
In this paper, we combine the concepts of interval-valued neutrosophic soft set and graph theory. We introduce notations of interval-valued neutrosophic soft graph and complete interval-valued neutrosophic soft graph. We also present several different types operations including cartesian product, union and intersection on interval-valued neutrosophic soft graphs and investigate some properties of them.
\end{abstract}

Keywords: Interval-valued neutrosophic sets, interval-valued neutrosophic soft sets, interval-valued neutrosophic graphs, intervalvalued neutrosophic soft graphs.

\section{Introduction}

The concept of neutrosophic set which is a powerful mathematical tool for dealing with incomplete, indeterminate and inconsistent information was firstly proposed by Smarandache [1]. Neutrosophic sets are generalization of the theory of fuzzy sets [2], intuitionistic fuzzy sets [3] and interval-valued intuitionistic fuzzy sets [4]. The neutrosophic sets are characterized by a truth-membership function $T$, an indeterminacy-membership function $I$ and a falsity membership function $F$ independently, which are within the real standard or nonstandard unit interval $]^{-} 0,1^{+}[$. Wang et al. [5] introduced the concept of a single-valued neutrosophic sets which is a subclass of the neutrosophic sets. They also introduced the concept of interval-valued neutrosophic sets [6] which is more sensitive than single valued neutrosophic sets in which three membership functions are independent and their value belong to the unit interval $[0,1]$. Some more work on single valued neutrosophic sets, neutrosophic sets, interval valued neutrosophic sets and their applications may be found on $[7,8,9,10,11,12]$.

The concept of soft set theory which is a new mathematical tool for dealing with uncertainties was initiated by Molodtsov [13]. It has been showed that soft sets have potential applications in different fields. Maji et al. [14] presented the definition of fuzzy soft sets and investigated some properties of this notion. Thereafter many researchers have applied this concept on different branches of mathematics. The concept of interval-valued fuzzy soft set has been introduced by Yang et al. [15]. The definition of neutrosophic soft sets was firstly given by Maji [16]. He also discussed many operations such as union, intersection and complement on neutrosophic soft sets. The concept of interval-valued neutrosophic soft set which is a generalization of fuzzy soft sets, interval-valued fuzzy soft sets and neutrosophic soft sets were given by Deli [17].

Graph theory was firstly introduced by Euler [18]. Since then graph theory has become the most important part of combinatorial mathematics. A graph is used to create a relationship between a given set of elements. Each element can be represented by a vertex and the relationship between them can be represented by an edge. The concept of graph has been applied to different algebraic structures in mathematics.

In this paper, primarily, we give the concepts of soft set, neutrosophic set, neutrosophic soft set, interval-valued neutrosophic soft set, graph and interval-valued neutrosophic graph. After that we introduce the notion of interval-valued 
neutrosophic soft graph and give some related examples. We also present several different types operations including cartesian product, union and intersection on interval-valued neutrosophic soft graphs and investigate some properties of them.

\section{Preliminaries}

Definition 1. [1] A neutrosophic set $A$ on the universe of discourse $U$ is defined as $A=\left\{\left\langle x, T_{A}(x), I_{A}(x)\right.\right.$, $\left.\left.F_{A}(x)\right\rangle, x \in U\right\}$, where the functions $\left.T, I, F: U \rightarrow\right]^{-} 0,1^{+}[$define a truth-membership function, an indeterminacy-membership function and a falsity-membership function of an element $x \in U$ for a set A, respectively, with the condition ${ }^{-} 0 \leq T_{A}(x)+I_{A}(x)+F_{A}(x) \leq 3^{+}$. The neutrosophic set takes the value from real standard or non-standard subsets of $]^{-} 0,1^{+}$. We consider the neutrosophic set which takes the value from the subset of $[0,1]$. The family of all neutrosphic sets on $U$ is denoted by $\mathscr{N}(U)$.

Definition 2. [16] Let $U$ be an inital universe set, $E$ be a set of parameters and $A \subseteq E$. If $F$ is a mapping given by $F: A \rightarrow \mathscr{N}(U)$, then a pair $(F, A)$ is called a neutrosophic soft set over $U$.

Definition 3. [17] Let $U$ be an inital universe set. Then an interval-valued neutrosophic set A over $U$ can be represented by the set $A=\left\{\left\langle x, T_{A}(x), I_{A}(x), F_{A}(x)\right\rangle, x \in U\right\}$, where

$$
\begin{aligned}
& T_{A}(x)=\left[T_{A}(x)^{-}, T_{A}(x)^{+}\right] \\
& I_{A}(x)=\left[I_{A}(x)^{-}, I_{A}(x)^{+}\right] \\
& F_{A}(x)=\left[F_{A}(x)^{-}, F_{A}(x)^{+}\right]
\end{aligned}
$$

and $0 \leq T_{A}(x)+I_{A}(x)+F_{A}(x) \leq 3$ for each point $x$ in $U$. The family of all interval-valued neutrosphic sets on $U$ is denoted by $I V \mathscr{N}(U)$.

Definition 4. [17] Let $U$ be an initial universe set and $A \subseteq E$ be a set of parameters. If $F$ is a mapping given by $F: A \rightarrow$ $\operatorname{IV} \mathscr{N}(U)$, then a pair $(F, A)$ is called an interval-valued neutrosophic soft set over $U$.

Definition 5. [18] A graph $G^{*}$ consists of set of finite objects $V=\left\{v_{1}, v_{2}, v_{3}, \ldots, v_{n}\right\}$ called vertices and other set $E=$ $\left\{e_{1}, e_{2}, e_{3}, \ldots, e_{n}\right\}$ whose elements are called edges. Usually a graph is denoted as $G^{*}=(V, E)$.

Definition 6. [19] An interval-valued neutrosophic graph of a graph $G^{*}=(V, E)$ is given by a pair $G=(A, B)$, where $A=<\left[T_{A}^{-}, T_{A}^{+}\right],\left[I_{A}^{-}, I_{A}^{+}\right],\left[F_{A}^{-}, F_{A}^{+}\right]>$is an interval-valued neutrosophic set on $V$ and $B=<\left[T_{B}^{-}, T_{B}^{+}\right],\left[I_{B}^{-}, I_{B}^{+}\right],\left[F_{B}^{-}, F_{A}^{+}\right]>$ is an interval-valued neutrosophic relation on $E$ such that

$$
\begin{gathered}
T_{B}^{-}\left(v_{i}, v_{j}\right) \leq \min \left\{T_{A}^{-}\left(v_{i}\right), T_{A}^{-}\left(v_{j}\right)\right\}, \quad T_{B}^{+}\left(v_{i}, v_{j}\right) \leq \min \left\{T_{A}^{+}\left(v_{i}\right), T_{A}^{+}\left(v_{j}\right)\right\} \\
I_{B}^{-}\left(v_{i}, v_{j}\right) \geq \max \left\{I_{A}^{-}\left(v_{i}\right), I_{A}^{-}\left(v_{j}\right)\right\}, \quad I_{B}^{+}\left(v_{i}, v_{j}\right) \geq \max \left\{I_{A}^{+}\left(v_{i}\right), I_{A}^{-}\left(v_{j}\right)\right\} \\
F_{B}^{-}\left(v_{i}, v_{j}\right) \geq \max \left\{F_{A}^{-}\left(v_{i}\right), F_{A}^{-}\left(v_{j}\right)\right\}, \quad F_{B}^{+}\left(v_{i}, v_{j}\right) \geq \max \left\{F_{A}^{+}\left(v_{i}\right), F_{A}^{+}\left(v_{j}\right)\right\}
\end{gathered}
$$

for all $\left(v_{i}, v_{j}\right) \in E$.

\section{Interval-valued neutrosophic soft graphs}

Definition 7. An interval-valued neutrosophic soft graph is given by ordered 4-tuple $G=\left(G^{*}, K, M, A\right)$ such that

(i) $G^{*}=(V, E)$ is a simple graph

(ii) $A$ is a nonempty set of parameters

(iii) $(K, A)$ is an interval-valued neutrosophic soft set over $V$

(iv) $(M, A)$ is an interval-valued neutrosophic soft set over $E$

(v) $(K(e), M(e))$ is an interval-valued neutrosophic graph of $G^{*}$ for all $e \in A$. That is

$$
T_{M(e)}^{-}(x, y) \leq \min \left\{T_{K(e)}^{-}(x), T_{K(e)}^{-}(y)\right\}, \quad T_{M(e)}^{+}(x y) \leq \min \left\{T_{K(e)}^{+}(x), T_{K(e)}^{+}(y)\right\}
$$




$$
\begin{array}{cc}
I_{M(e)}^{-}(x, y) \geq \max \left\{I_{K(e)}^{-}(x), I_{K(e)}^{-}(y)\right\}, & I_{M(e)}^{+}(x y) \geq \max \left\{I_{K(e)}^{+}(x), I_{K(e)}^{+}(y)\right\} \\
F_{M(e)}^{-}(x, y) \geq \max \left\{F_{K(e)}^{-}(x), F_{K(e)}^{-}(y)\right\}, & F_{M(e)}^{+}(x y) \geq \max \left\{F_{K(e)}^{+}(x), F_{K(e)}^{+}(y)\right\}
\end{array}
$$

such that $0 \leq T_{M(e)}(x, y)+I_{M(e)}(x, y)+F_{M(e)}(x, y) \leq 3$ for all $e \in A$ and $x, y \in V$. The interval-valued neutrosophic graph $(K(e), M(e))$ is denoted by $H(e)$ for convenience. An interval-valued neutrosophic soft graph is a parameterized family of interval-valued neutrosophic graphs.

Example 1. Consider a simple graph $G^{*}=(V, E)$ such that $V=\left\{v_{1}, v_{2}, v_{3}\right\}$ and $E=\left\{v_{1} v_{2}, v_{2} v_{3}, v_{3} v_{1}\right\}$. Let $A=\left\{e_{1}, e_{2}\right\}$ be a set of parameters and let $(K, A)$ be an interval-valued neutrosophic soft set over $V$ with its approximate function $K: A \rightarrow I V \mathscr{N}(V)$ defined by

$$
\begin{aligned}
& K\left(e_{1}\right)=\left\{v_{1}\left|([0.2,0.6],[0.1,0.3],[0.2,0.3]), v_{2}\right|\left([0.4,0.5],[0.6,0.7],[0.3,0.4], v_{3} \mid([0.2,0.3],[0.2,0.4],[0.3,0.5])\right\}\right. \\
& K\left(e_{2}\right)=\left\{v_{1}\left|([0.3,0.4],[0.4,0.5],[0.2,0.7]), v_{2}\right|([0.2,0.4],[0.3,0.4],[0.4,0.5]), v_{3} \mid([0.2,0.3],[0.5,0.6],[0.4,0.8])\right\}
\end{aligned}
$$

Now let $(M, A)$ be an interval-valued neutrosophic soft set over $E$ with its approximate function $M: A \rightarrow I V \mathscr{N}(E)$ defined by

$$
\begin{aligned}
& M\left(e_{1}\right)=\left\{v_{1} v_{2} \mid([0.1,0.4],[0.7,0.8],[0.4,0.5])\right\} \\
& M\left(e_{2}\right)=\left\{v_{1} v_{2}\left|([0.1,0.2],[0.6,0.7],[0.5,0.8]), v_{2} v_{3}\right|([0.1,0.2],[0.6,0.7],[0.6,0.9]), v_{3} v_{1} \mid([0.1,0.3],[0.6,0.7],[0.5,0.8])\right\}
\end{aligned}
$$

It is clearly seen that $H\left(e_{1}\right)=\left(K\left(e_{1}\right), M\left(e_{1}\right)\right), H\left(e_{2}\right)=\left(K\left(e_{2}\right), M\left(e_{2}\right)\right)$ are interval-valued neutrosophic graphs corresponding to the parameters $e_{1}$ and $e_{2}$. Hence $G=\left(G^{*}, K, M, A\right)$ is an interval-valued neutrosophic soft graph of $G^{*}$ as shown in Figure 1.
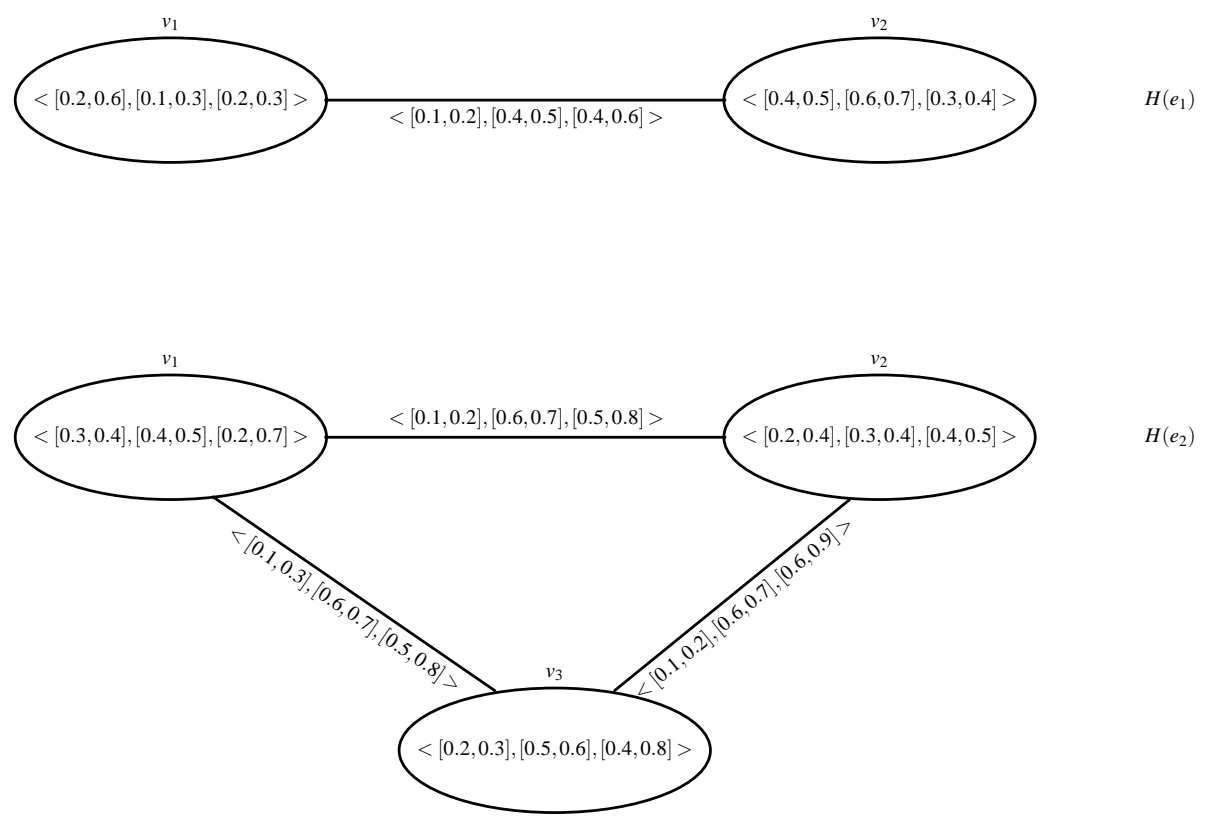

Fig. 1: Interval-valued neutrosophic soft graph $G$.

Definition 8. Let $G_{1}=\left(G^{*}, K_{1}, M_{1}, A\right)$ and $G_{2}=\left(G^{*}, K_{2}, M_{2}, B\right)$ be two interval-valued neutrosophic soft graphs of $G^{*}$. Then $G_{1}$ is called an interval-valued neutrosophic soft subgraph of $G_{2}$ if

(i) $A \subseteq B$

(ii) $H_{1}(e)=\left(K_{1}(e), M_{1}(e)\right)$ is an interval-valued neutrosophic subgraph of $H_{2}(e)=\left(K_{2}(e), M_{2}(e)\right)$ for all $e \in A$. 
Example 2. Consider the simple graph $G^{*}=(V, E)$ as taken in Example 1.

Now let $B=\left\{e_{1}\right\}$ be a parameter set, $\left(K_{1}, B\right)$ be an interval-valued neutrosophic soft set over $V$ and $\left(M_{1}, B\right)$ be an intervalvalued neutrosophic soft set on $E$ defined by

$$
\begin{aligned}
K_{1}\left(e_{1}\right) & =\left\{v_{1}\left|([0.1,0.4],[0.3,0.5],[0.4,0.6]), v_{2}\right|\left([0.3,0.4],[0.7,0.8],[0.5,0.7], v_{3} \mid([0.1,0.3],[0.4,0.5],[0.6,0.7])\right\}\right. \\
M_{1}\left(e_{1}\right) & =\left\{v_{1} v_{2}\left|([0.1,0.3],[0.8,0.9],[0.7,0.9]), v_{2} v_{3}\right|([0.1,0.2],[0.8,0.9],[0.7,0.8]), v_{3} v_{1} \mid([0.1,0.2],[0.5,0.6],[0.7,0.8])\right\}
\end{aligned}
$$

It is clearly seen that $H_{1}\left(e_{1}\right)=\left(K_{1}\left(e_{1}\right), M_{1}\left(e_{1}\right)\right)$ is interval-valued neutrosophic graphs corresponding to the parameters $e_{1}$. Also $G_{1}=\left(G^{*}, K_{1}, M_{1}, B\right)$ is an interval-valued neutrosophic soft graph as shown in Figure 2 . Hence $G_{1}$ is an intervalvalued neutrosophic soft subgraph of $G$.

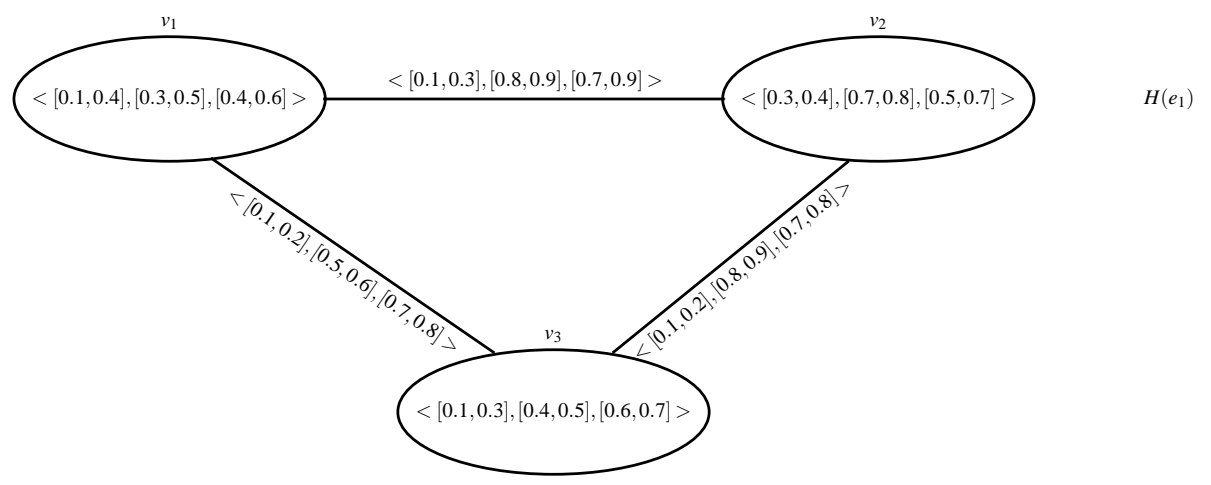

Fig. 2: Interval-valued neutrosophic soft graph $G_{1}$

Definition 9. An interval-valued neutrosophic soft graph $G=\left(G^{*}, K, M, A\right)$ is called a complete interval-valued neutrosophic soft graph if

$$
\begin{aligned}
T_{M(e)}^{-}(x, y) & =\min \left\{T_{K(e)}^{-}(x), T_{K(e)}^{-}(y)\right\} \\
T_{M(e)}^{+}(x, y) & =\min \left\{T_{K(e)}^{+}(x), T_{K(e)}^{+}(y)\right\} \\
I_{M(e)}^{-}(x, y) & =\max \left\{I_{K(e)}^{-}(x), I_{K(e)}^{-}(y)\right\} \\
I_{M(e)}^{+}(x, y) & =\max \left\{I_{K(e)}^{+}(x), I_{K(e)}^{+}(y)\right\} \\
F_{M(e)}^{-}(x, y) & =\max \left\{F_{K(e)}^{-}(x), F_{K(e)}^{-}(y)\right\} \\
F_{M(e)}^{+}(x, y) & =\max \left\{F_{K(e)}^{+}(x), F_{K(e)}^{+}(y)\right\}
\end{aligned}
$$

for all $e \in A$ and $x y \in E$.

Example 3. Consider a simple graph $G^{*}=(V, E)$ such that

$$
V=\left\{v_{1}, v_{2}, v_{3}, v_{4}\right\} \text { and } E=\left\{v_{1} v_{2}, v_{2} v_{3}, v_{3} v_{4}, v_{4} v_{1}, v_{1} v_{3}, v_{2} v_{4}\right\}
$$

Let $A=\left\{e_{1}, e_{2}\right\}$ be a set of parameters and $(K, A)$ be an interval-valued neutrosophic soft sets over $V$ with its approximation function $K: A \rightarrow I V \mathscr{N}(V)$ defined by

$$
\begin{aligned}
K\left(e_{1}\right)= & \left\{v_{1}\left|([0.2,0.3],[0.1,0.5],[0.5,0.6]), v_{2}\right|([0.1,0.4],[0.3,0.4],[0.4,0.7]),\right. \\
& \left.v_{3}\left|([0.1,0.2],[0.1,0.3],[0.5,0.7]), v_{4}\right|([0.4,0.5],[0.3,0.5],[0.6,0.8])\right\} \\
K\left(e_{2}\right)= & \left\{v_{1}\left|([0.3,0.4],[0.2,0.5],[0.7,0.9]), v_{2}\right|([0.1,0.2],[0.3,0.4],[0.4,0.7]),\right. \\
& \left.v_{3} \mid([0.2,0.4],[0.3,0.4],[0.5,0.7])\right\}, v_{4} \mid([0.5,0.7],[0.2,0.3],[0.4,0.6])
\end{aligned}
$$


Let $(M, A)$ be an interval-valued neutrosophic soft sets over $E$ with its approximation function $K: A \rightarrow I V \mathscr{N}(E)$ defined by

$$
\begin{aligned}
M\left(e_{1}\right)= & \left\{v_{1} v_{2}\left|([0.1,0.3],[0.3,0.5],[0.5,0.7]), v_{2} v_{3}\right|([0.1,0.2],[0.3,0.4],[0.5,0.7]), v_{3} v_{1} \mid([0.1,0.2],[0.1,0.5],[0.5,0.7])\right\} \\
M\left(e_{2}\right)=\{ & \left\{v_{1} v_{2}\left|([0.1,0.2],[0.3,0.5],[0.7,0.9]), v_{2} v_{3}\right|([0.1,0.2],[0.3,0.4],[0.5,0.7]), v_{3} v_{4} \mid([0.2,0.4],[0.3,0.4],[0.5,0.7]),\right. \\
& \left.v_{4} v_{1}\left|([0.3,0.4],[0.2,0.5],[0.7,0.9]), v_{1} v_{3}\right|([0.2,0.4],[0.3,0.5],[0.7,0.9]), v_{2} v_{4} \mid([0.1,0.2],[0.3,0.4],[0.4,0.7])\right\}
\end{aligned}
$$

It is easy to see that $H\left(e_{1}\right)$ and $H\left(e_{2}\right)$ are interval valued neutrosophic graphs of $G^{*}$ corresponding to the parameters $e_{1}$, $e_{2}$ and $e_{3}$ respectively. Hence $G=\left(G^{*}, K, M, A\right)$ is a complete interval-valued neutrosophic soft graph of $G^{*}$.

Definition 10. Let $G_{1}=\left(G_{1}^{*}, K_{1}, M_{1}, A\right)$ and $G_{2}=\left(G_{2}^{*}, K_{2}, M_{2}, B\right)$ be two interval valued neutrosophic soft graphs of simple graphs $G_{1}^{*}=\left(V_{1}, E_{1}\right)$ and $G_{2}^{*}=\left(V_{2}, E_{2}\right)$, respectively. The cartesian product of $G_{1}$ and $G_{2}$ is denoted by $G_{1} \times G_{2}=$ $\left(G^{*}, K, M, A \times B\right)$, where $G^{*}=\left(V_{1} \times V_{2}, E_{1} \times E_{2}\right)$, and is defined by

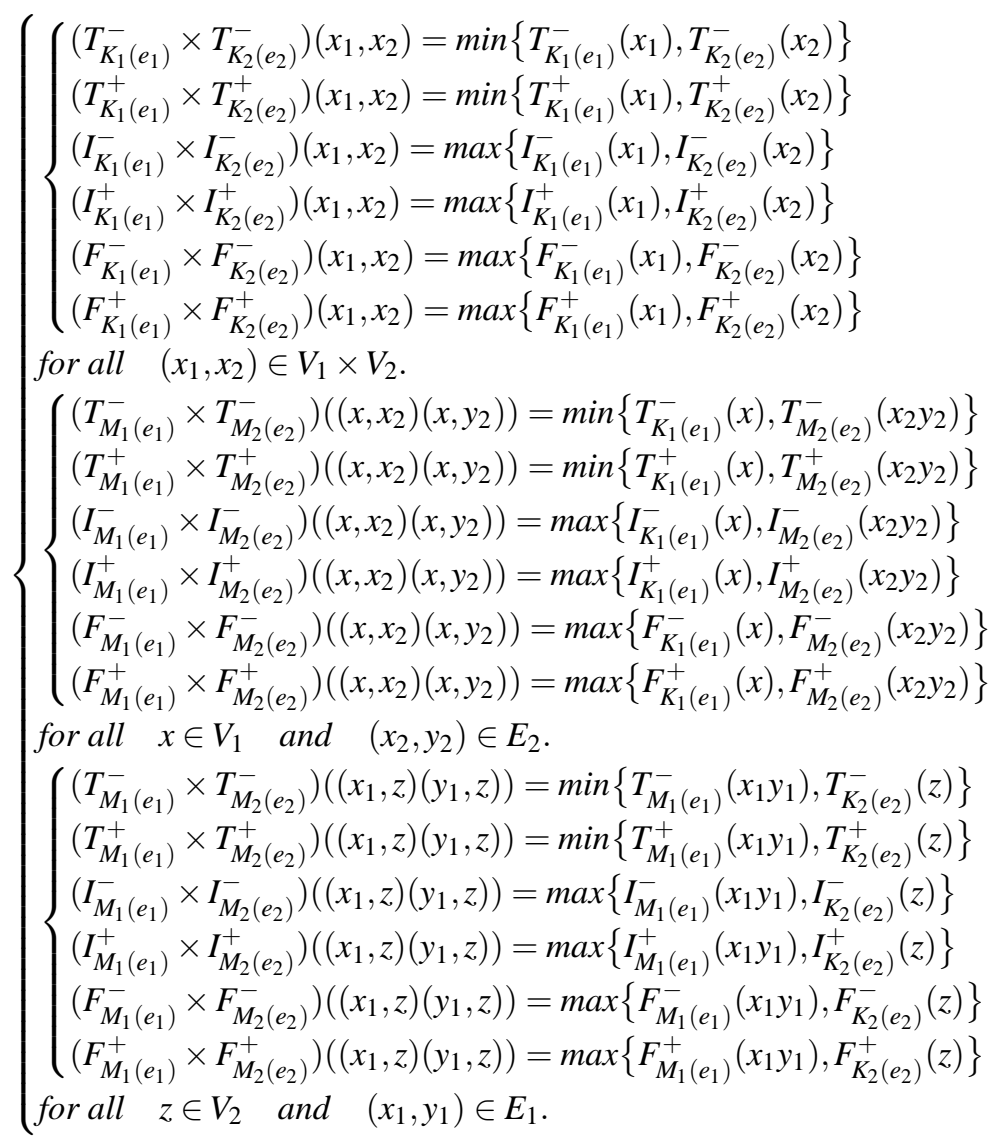

for all $e_{1} \in A$ and $e_{2} \in B$.

Example 4. Consider two graphs $G_{1}^{*}=\left(V_{1}, E_{1}\right)$ and $G_{2}^{*}=\left(V_{2}, E_{2}\right)$ such that

$$
V_{1}=\left\{u_{1}, u_{2}\right\}, E_{1}=\left\{u_{1} u_{2}\right\} \text { and } V_{2}=\left\{v_{1}, v_{2}, v_{3}\right\}, E_{2}=\left\{v_{1} v_{2}, v_{2} v_{3}\right\}
$$

Let $A=\left\{e_{1}\right\}$ be a set of parameters, and let $\left(K_{1}, A\right)$ and $\left(M_{1}, A\right)$ be two interval-valued neutrosophic soft sets over $V_{1}$ and $E_{1}$, respectively, defined by

$$
\begin{aligned}
K_{1}\left(e_{1}\right) & =\left\{u_{1}\left|([0.3,0.5],[0.1,0.2],[0.4,0.6]), u_{2}\right|([0.5,0.6],[0.2,0.4],[0.1,0.3])\right\} \\
M_{1}\left(e_{1}\right) & =\left\{u_{1} u_{2} \mid([0.2,0.3],[0.3,0.5],[0.6,0.7])\right\}
\end{aligned}
$$


Now let $B=\left\{e_{2}\right\}$ be a set of parameters, and let $\left(K_{2}, B\right)$ and $\left(M_{2}, B\right)$ be two interval-valued neutrosophic soft sets over $V_{2}$ and $E_{2}$, respectively, defined by

$$
\begin{aligned}
K_{2}\left(e_{2}\right) & =\left\{v_{1}\left|([0.4,0.5],[0.3,0.4],[0.6,0.7]), v_{2}\right|([0.3,0.6],[0.2,0.3],[0.1,0.4]), v_{3} \mid([0.2,0.5],[0.4,0.6],[0.3,0.5])\right\} \\
M_{2}\left(e_{2}\right) & =\left\{v_{1} v_{2}\left|([0.1,0.2],[0.4,0.5],[0.7,0.8]), v_{2} v_{3}\right|([0.1,0.4],[0.6,0.7],[0.8,0.9])\right\}
\end{aligned}
$$

It is easy to see that, $H\left(e_{1}\right)=\left(K_{1}\left(e_{1}\right), M_{1}\left(e_{1}\right)\right)$ and $H\left(e_{2}\right)=\left(K_{2}\left(e_{2}\right), M_{2}\left(e_{2}\right)\right)$ are interval-valued neutrosophic graphs. Hence $G_{1}=\left(G_{1}^{*}, K_{1}, M_{1}, A\right)$ and $G_{2}=\left(G_{2}^{*}, K_{2}, M_{2}, B\right)$ are interval-valued neutrosophic soft graphs of $G_{1}^{*}$ and $G_{2}^{*}$, respectively, as shown in Figure 3.
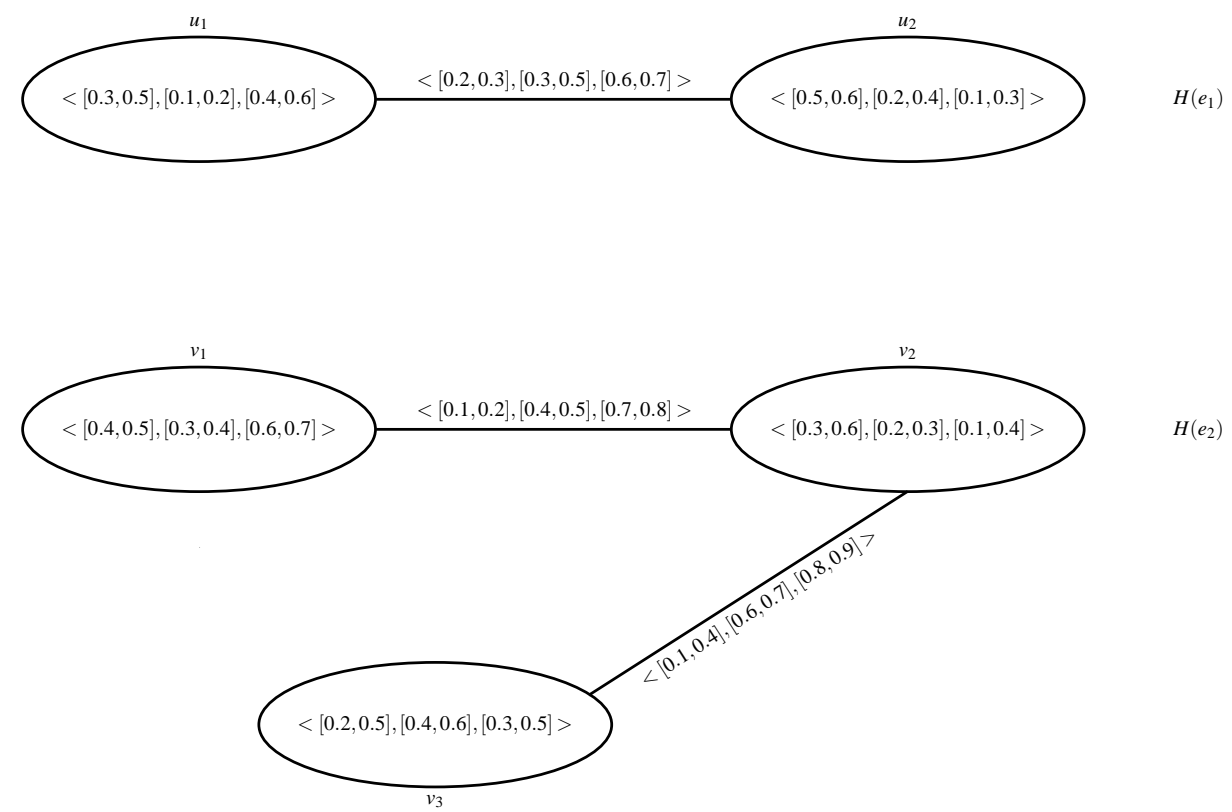

Fig. 3: Interval-valued neutrosophic soft graph $G_{1}$ and $G_{2}$

The cartesian product of $G_{1}$ and $G_{2}$ is as shown in Figure 4.

Theorem 1. If $G_{1}$ and $G_{2}$ are two interval-valued neutrosophic soft graphs, then so is $G_{1} \times G_{2}$.

Proof. Let $G_{1}=\left(G_{1}^{*}, K_{1}, M_{1}, A\right)$ and $G_{2}=\left(G_{2}^{*}, K_{2}, M_{2}, B\right)$ be two interval-valued neutrosophic soft graphs of $G_{1}^{*}=\left(V_{1}, E_{1}\right)$ and $G_{2}^{*}=\left(V_{2}, E_{2}\right)$, respectively. From Definition 10, for all $e_{1} \in A$ and $e_{2} \in B$, there are three cases.

Case (i) If $x_{1} \in V_{1}$ and $x_{2} \in V_{2}$, then

$$
\left(T_{K_{1}\left(e_{1}\right)}^{-} \times T_{K_{2}\left(e_{2}\right)}^{-}\right)_{\left(x_{1}, x_{2}\right)}=\min \left(T_{K_{1}\left(e_{1}\right)}^{-}\left(x_{1}\right), T_{K_{2}\left(e_{2}\right)}^{-}\left(x_{2}\right)\right) \leq \min \left[\left(T_{K_{1}\left(e_{1}\right)}^{-} \times T_{K_{2}\left(e_{2}\right)}^{-}\right)_{\left(x_{1}\right)},\left(T_{K_{1}\left(e_{1}\right)}^{-} \times T_{K_{2}\left(e_{2}\right)}^{-}\right)_{\left(x_{2}\right)}\right]
$$

Similarly, we can show that $\left(T_{K_{1}\left(e_{1}\right)}^{+} \times T_{K_{2}\left(e_{2}\right)}^{+}\right)_{\left(x_{1}, x_{2}\right)} \leq \min \left[\left(T_{K_{1}\left(e_{1}\right)}^{+} \times T_{K_{2}\left(e_{2}\right)}^{+}\right)_{\left(x_{1}\right)},\left(T_{K_{1}\left(e_{1}\right)}^{+} \times T_{K_{2}\left(e_{2}\right)}^{+}\right)_{\left(x_{2}\right)}\right]$

$$
\left(I_{K_{1}\left(e_{1}\right)}^{-} \times I_{K_{2}\left(e_{2}\right)}^{-}\right)_{\left(x_{1}, x_{2}\right)}=\max \left(I_{K_{1}\left(e_{1}\right)}^{-}\left(x_{1}\right), I_{K_{2}\left(e_{2}\right)}^{-}\left(x_{2}\right)\right) \geq \max \left[\left(I_{K_{1}\left(e_{1}\right)}^{-} \times I_{K_{2}\left(e_{2}\right)}^{-}\right)_{\left(x_{1}\right)},\left(I_{K_{1}\left(e_{1}\right)}^{-} \times I_{K_{2}\left(e_{2}\right)}^{-}\right)_{\left(x_{2}\right)}\right]
$$

Similarly, we can show that $\left(I_{K_{1}\left(e_{1}\right)}^{+} \times I_{K_{2}\left(e_{2}\right)}^{+}\right)_{\left(x_{1}, x_{2}\right)} \geq \max \left[\left(I_{K_{1}\left(e_{1}\right)}^{+} \times I_{K_{2}\left(e_{2}\right)}^{+}\right)_{\left(x_{1}\right)},\left(I_{K_{1}\left(e_{1}\right)}^{+} \times I_{K_{2}\left(e_{2}\right)}^{+}\right)_{\left(x_{2}\right)}\right]$

$$
\left(F_{K_{1}\left(e_{1}\right)}^{-} \times F_{K_{2}\left(e_{2}\right)}^{-}\right)_{\left(x_{1}, x_{2}\right)}=\max \left(F_{K_{1}\left(e_{1}\right)}^{-}\left(x_{1}\right), F_{K_{2}\left(e_{2}\right)}^{-}\left(x_{2}\right)\right) \geq \max \left[\left(F_{K_{1}\left(e_{1}\right)}^{-} \times F_{K_{2}\left(e_{2}\right)}^{-}\right)_{\left(x_{1}\right)},\left(F_{K_{1}\left(e_{1}\right)}^{-} \times F_{K_{2}\left(e_{2}\right)}^{-}\right)_{\left(x_{2}\right)}\right]
$$




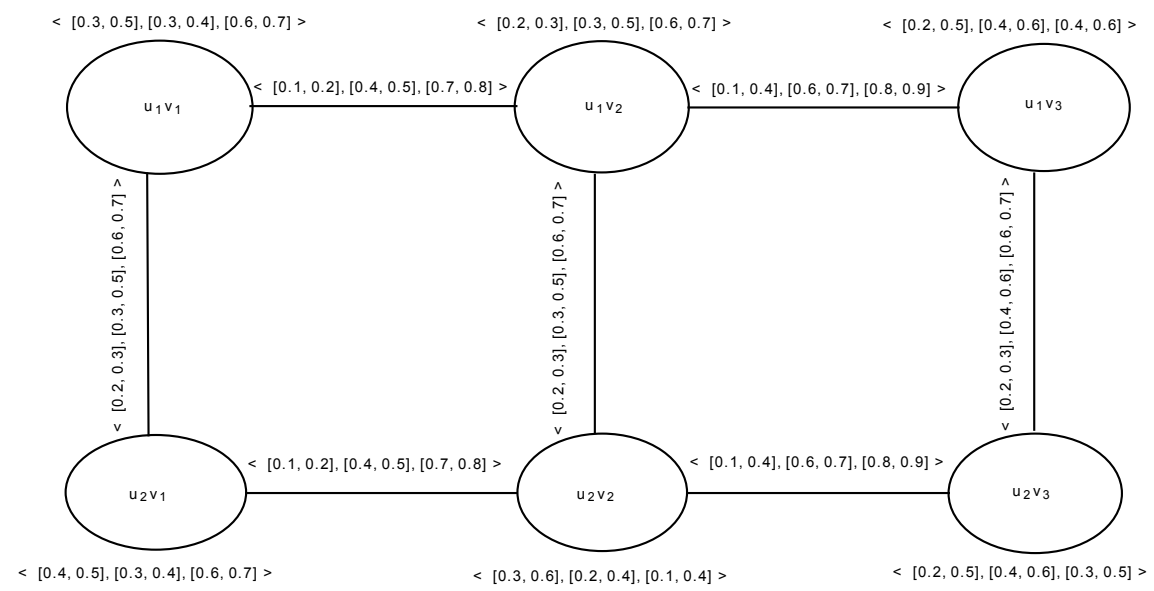

Fig. 4: Cartesian product of $G_{1}$ and $G_{2}$

Similarly, we can show that $\left(F_{K_{1}\left(e_{1}\right)}^{+} \times F_{K_{2}\left(e_{2}\right)}^{+}\right)_{\left(x_{1}, x_{2}\right)} \geq \max \left[\left(F_{K_{1}\left(e_{1}\right)}^{+} \times F_{K_{2}\left(e_{2}\right)}^{+}\right)_{\left(x_{1}\right)},\left(F_{K_{1}\left(e_{1}\right)}^{+} \times F_{K_{2}\left(e_{2}\right)}^{+}\right)_{\left(x_{2}\right)}\right]$

Case(ii) If $x \in V_{1}$ and $\left(x_{2}, y_{2}\right) \in E_{2}$, then

$$
\begin{aligned}
\left(T_{M_{1}\left(e_{1}\right)}^{-} \times T_{M_{2}\left(e_{2}\right)}^{-}\right)_{\left(\left(x, x_{2}\right)\left(x, y_{2}\right)\right)} & =\min \left(T_{K_{1}\left(e_{1}\right)}^{-}(x), T_{M_{2}\left(e_{2}\right)}^{-}\left(x_{2}, y_{2}\right)\right) \\
& \leq \min \left[T_{K_{1}\left(e_{1}\right)}^{-}(x), \min \left(T_{K_{2}\left(e_{2}\right)}^{-}\left(x_{2}\right), T_{K_{2}\left(e_{2}\right)}^{-}\left(y_{2}\right)\right)\right] \\
& =\min \left[\min \left(T_{K_{1}\left(e_{1}\right)}^{-}(x), T_{K_{2}\left(e_{2}\right)}^{-}\left(x_{2}\right)\right), \min \left(T_{K_{1}\left(e_{1}\right)}^{-}(x), T_{K_{2}\left(e_{2}\right)}^{-}\left(y_{2}\right)\right)\right] \\
& =\min \left[\left(T_{K_{1}\left(e_{1}\right)}^{-} \times T_{K_{2}\left(e_{2}\right)}^{-}\right)_{\left(x, x_{2}\right)},\left(T_{K_{1}\left(e_{1}\right)}^{-} \times T_{K_{2}\left(e_{2}\right)}^{-}\right)_{\left(x, y_{2}\right)}\right]
\end{aligned}
$$

Similarly, we can show that $\left(T_{M_{1}\left(e_{1}\right)}^{+} \times T_{M_{2}\left(e_{2}\right)}^{+}\right)_{\left(\left(x, x_{2}\right)\left(x, y_{2}\right)\right)} \leq \min \left[\left(T_{K_{1}\left(e_{1}\right)}^{+} \times T_{K_{2}\left(e_{2}\right)}^{+}\right)_{\left(x, x_{2}\right)},\left(T_{K_{1}\left(e_{1}\right)}^{+} \times T_{K_{2}\left(e_{2}\right)}^{+}\right)_{\left(x, y_{2}\right)}\right]$

$$
\begin{aligned}
\left(I_{M_{1}\left(e_{1}\right)}^{-} \times I_{M_{2}\left(e_{2}\right)}^{-}\right)_{\left(\left(x, x_{2}\right)\left(x, y_{2}\right)\right)} & =\max \left(I_{K_{1}\left(e_{1}\right)}^{-}(x), I_{M_{2}\left(e_{2}\right)}^{-}\left(x_{2}, y_{2}\right)\right) \\
& \geq \max \left[I_{K_{1}\left(e_{1}\right)}^{-}(x), \max \left(I_{K_{2}\left(e_{2}\right)}^{-}\left(x_{2}\right), I_{K_{2}\left(e_{2}\right)}^{-}\left(y_{2}\right)\right)\right] \\
& =\max \left[\max \left(I_{K_{1}\left(e_{1}\right)}^{-}(x), I_{K_{2}\left(e_{2}\right)}^{-}\left(x_{2}\right)\right), \max \left(I_{K_{1}\left(e_{1}\right)}^{-}(x), I_{K_{2}\left(e_{2}\right)}^{-}\left(y_{2}\right)\right)\right] \\
& =\max \left[\left(I_{K_{1}\left(e_{1}\right)}^{-} \times I_{K_{2}\left(e_{2}\right)}^{-}\right)_{\left(x, x_{2}\right)},\left(I_{K_{1}\left(e_{1}\right)}^{-} \times I_{K_{2}\left(e_{2}\right)}^{-}\right)_{\left(x, y_{2}\right)}\right]
\end{aligned}
$$

Similarly, we can show that $\left(I_{M_{1}\left(e_{1}\right)}^{+} \times I_{M_{2}\left(e_{2}\right)}^{+}\right)_{\left(\left(x, x_{2}\right)\left(x, y_{2}\right)\right)} \geq \max \left[\left(I_{K_{1}\left(e_{1}\right)}^{+} \times I_{K_{2}\left(e_{2}\right)}^{+}\right)_{\left(x, x_{2}\right)},\left(I_{K_{1}\left(e_{1}\right)}^{+} \times I_{K_{2}\left(e_{2}\right)}^{+}\right)_{\left(x, y_{2}\right)}\right]$

$$
\begin{aligned}
\left(F_{M_{1}\left(e_{1}\right)}^{-} \times F_{M_{2}\left(e_{2}\right)}^{-}\right)_{\left(\left(x, x_{2}\right)\left(x, y_{2}\right)\right)} & =\max \left(F_{K_{1}\left(e_{1}\right)}^{-}(x), F_{M_{2}\left(e_{2}\right)}^{-}\left(x_{2}, y_{2}\right)\right) \\
& \geq \max \left[I_{K_{1}\left(e_{1}\right)}^{-}(x), \max \left(F_{K_{2}\left(e_{2}\right)}^{-}\left(x_{2}\right), F_{K_{2}\left(e_{2}\right)}^{-}\left(y_{2}\right)\right)\right] \\
& =\max \left[\max \left(F_{K_{1}\left(e_{1}\right)}^{-}(x), F_{K_{2}\left(e_{2}\right)}^{-}\left(x_{2}\right)\right), \max \left(F_{K_{1}\left(e_{1}\right)}^{-}(x), F_{K_{2}\left(e_{2}\right)}^{-}\left(y_{2}\right)\right)\right] \\
& =\max \left[\left(F_{K_{1}\left(e_{1}\right)}^{-} \times F_{K_{2}\left(e_{2}\right)}^{-}\right)_{\left(x, x_{2}\right)},\left(F_{K_{1}\left(e_{1}\right)}^{-} \times F_{K_{2}\left(e_{2}\right)}^{-}\right)_{\left(x, y_{2}\right)}\right]
\end{aligned}
$$

Similarly, we can show that $\left(F_{M_{1}\left(e_{1}\right)}^{+} \times F_{M_{2}\left(e_{2}\right)}^{+}\right)_{\left(\left(x, x_{2}\right)\left(x, y_{2}\right)\right)} \geq \max \left[\left(F_{K_{1}\left(e_{1}\right)}^{+} \times F_{K_{2}\left(e_{2}\right)}^{+}\right)_{\left(x, x_{2}\right)},\left(F_{K_{1}\left(e_{1}\right)}^{+} \times F_{K_{2}\left(e_{2}\right)}^{+}\right)_{\left(x, y_{2}\right)}\right]$

Case (iii) If $x \in V_{2}$ and $\left(x_{1}, y_{1}\right) \in E_{1}$, then it can be shown in a similar way to case (ii).

Definition 11. Let $G_{1}=\left(G_{1}^{*}, K_{1}, M_{1}, A\right)$ and $G_{2}=\left(G_{2}^{*}, K_{2}, M_{2}, B\right)$ be two interval-valued neutrosophic soft graphs of simple graphs $G_{1}^{*}=\left(V_{1}, E_{1}\right)$ and $G_{2}^{*}=\left(V_{2}, E_{2}\right)$, respectively. The union of $G_{1}$ and $G_{2}$ is denoted by $G_{1} \cup G_{2}=\left(G^{*}, K, M, A \cup B\right)$, where $G^{*}=\left(V_{1} \cap V_{2}, E_{1} \cap E_{2}\right)$, and is defined by 


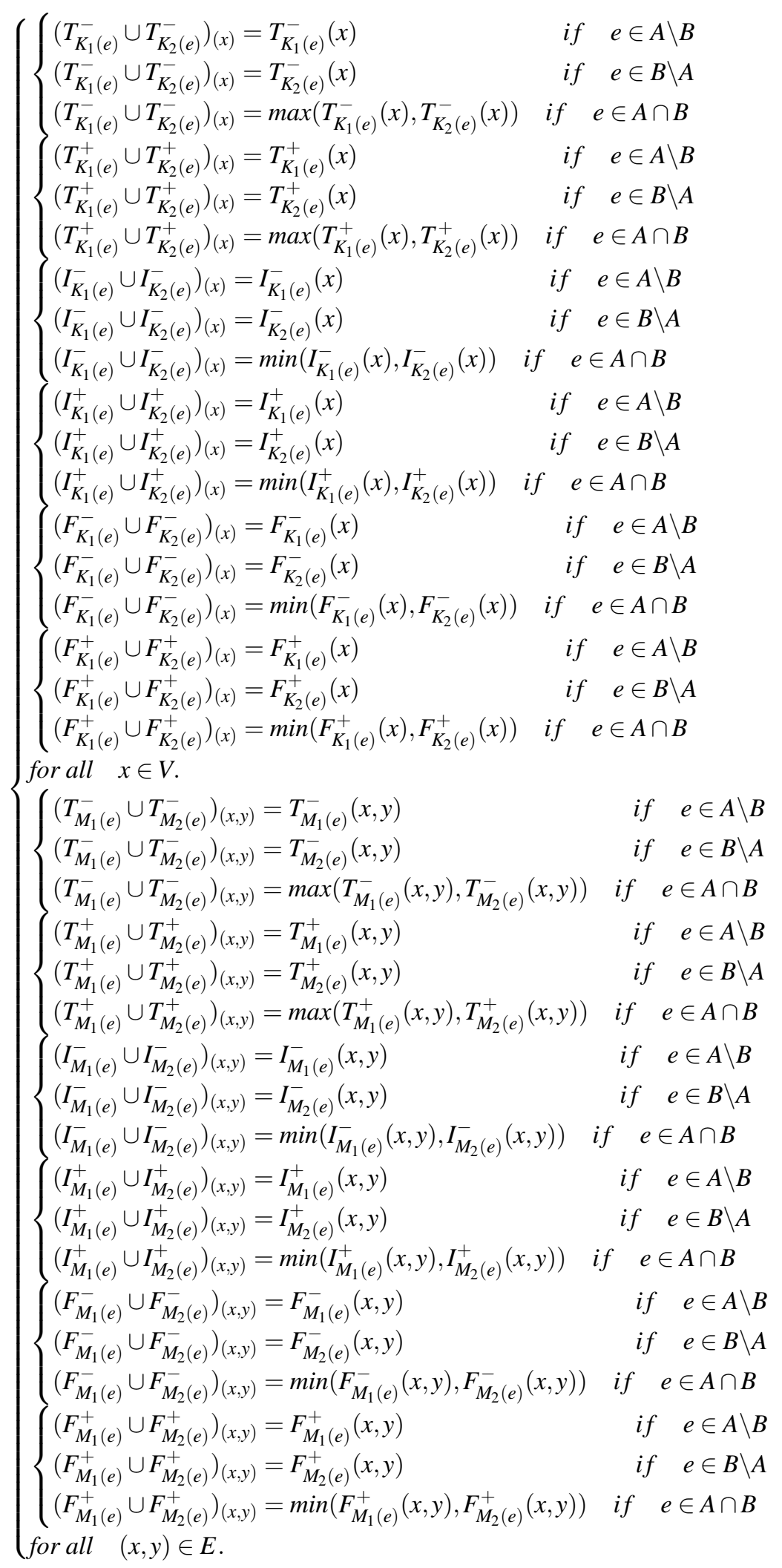

Example 5. Consider two graphs $G_{1}^{*}=\left(V_{1}, E_{1}\right)$ and $G_{2}^{*}=\left(V_{2}, E_{2}\right)$ such that $V_{1}=\left\{u_{1}, u_{2}, u_{3}\right\}, E_{1}=\left\{u_{1} u_{2}, u_{1} u_{3}, u_{2} u_{3}\right\}$ and $V_{2}=\left\{v_{1}, v_{2}\right\}, E_{2}=\left\{u_{1}, u_{3}, v_{1} v_{2}\right\}$. Let $A=\left\{e_{1}, e_{2}\right\}$ be a set of parameters and let $\left(K_{1}, A\right)$ and $\left(M_{1}, A\right)$ be two interval-valued 
neutrosophic soft sets over $V_{1}$ and $E_{1}$ respectively, defined by

$$
\begin{aligned}
K_{1}\left(e_{1}\right) & =\left\{u_{1}\left|([0.4,0.6],[0.2,0.3],[0.1,0.3]), u_{2}\right|([0.4,0.7],[0.2,0.4],[0.1,0.3])\right\} \\
M_{1}\left(e_{1}\right) & =\left\{u_{1} u_{2} \mid([0.3,0.4],[0.4,0.5],[0.3,0.5])\right\} \\
K_{1}\left(e_{2}\right) & =\left\{u_{1}\left|([0.3,0.5],[0.2,0.3],[0.3,0.4]), u_{2}\right|([0.2,0.3],[0.2,0.3],[0.1,0.4]), u_{3} \mid([0.1,0.3],[0.2,0.4],[0.3,0.5])\right\} \\
M_{1}\left(e_{2}\right) & =\left\{u_{1} u_{3}\left|([0.1,0.2],[0.3,0.5],[0.4,0.6]), u_{2} u_{3}\right|([0.1,0.3],[0.4,0.5],[0.4,0.5])\right\}
\end{aligned}
$$

Now let $B=\left\{e_{2}, e_{3}\right\}$ be a set of parameters and let $\left(K_{2}, A\right)$ and $\left(M_{2}, A\right)$ be two interval-valued neutrosophic soft sets over $V_{2}$ and $E_{2}$ respectively, defined by

$$
\begin{aligned}
K_{2}\left(e_{2}\right) & =\left\{v_{1}\left|([0.2,0.3],[0.1,0.2],[0.3,0.5]), v_{2}\right|([0.2,0.4],[0.1,0.3],[0.2,0.5])\right\} \\
M_{2}\left(e_{2}\right) & =\left\{v_{1} v_{2} \mid([0.1,0.2],[0.4,0.5],[0.4,0.6])\right\} \\
K_{2}\left(e_{3}\right) & =\left\{v_{1}\left|([0.3,0.5],[0.2,0.3],[0.3,0.4]), v_{2}\right|([0.2,0.3],[0.1,0.3],[0.4,0.5]\}\right. \\
M_{2}\left(e_{3}\right) & =\left\{v_{1} v_{2} \mid([0.1,0.2],[0.3,0.4],[0.4,0.5]\}\right.
\end{aligned}
$$

It is easy to see that $H_{1}\left(e_{1}\right)=\left(K_{1}\left(e_{1}\right), M_{1}\left(e_{1}\right)\right), H_{1}\left(e_{2}\right)=\left(K_{1}\left(e_{2}\right), M_{1}\left(e_{2}\right)\right), H_{2}\left(e_{2}\right)=\left(K_{2}\left(e_{2}\right), M_{2}\left(e_{2}\right)\right)$ and $H_{2}\left(e_{3}\right)=\left(K_{2}\left(e_{3}\right), M_{2}\left(e_{3}\right)\right)$ are interval valued neutrosophic graphs. Hence $G_{1}=\left(G_{1}^{*}, K_{1}, M_{1}, A\right)$ and $G_{2}=\left(G_{2}^{*}, K_{2}, M_{2}, B\right)$ are interval-valued neutrosophic soft graphs of $G_{1}^{*}$ and $G_{2}^{*}$ respectively as shown in Figure 5 and Figure 6. From Definition 12, $G_{1} \cup G_{2}=\left(G^{*}, K, M, A \cup B\right)$, where $A \cup B=\left\{e_{1}, e_{2}, e_{3}\right\}$. Also $H\left(e_{1}\right)=H_{1}\left(e_{1}\right)$,
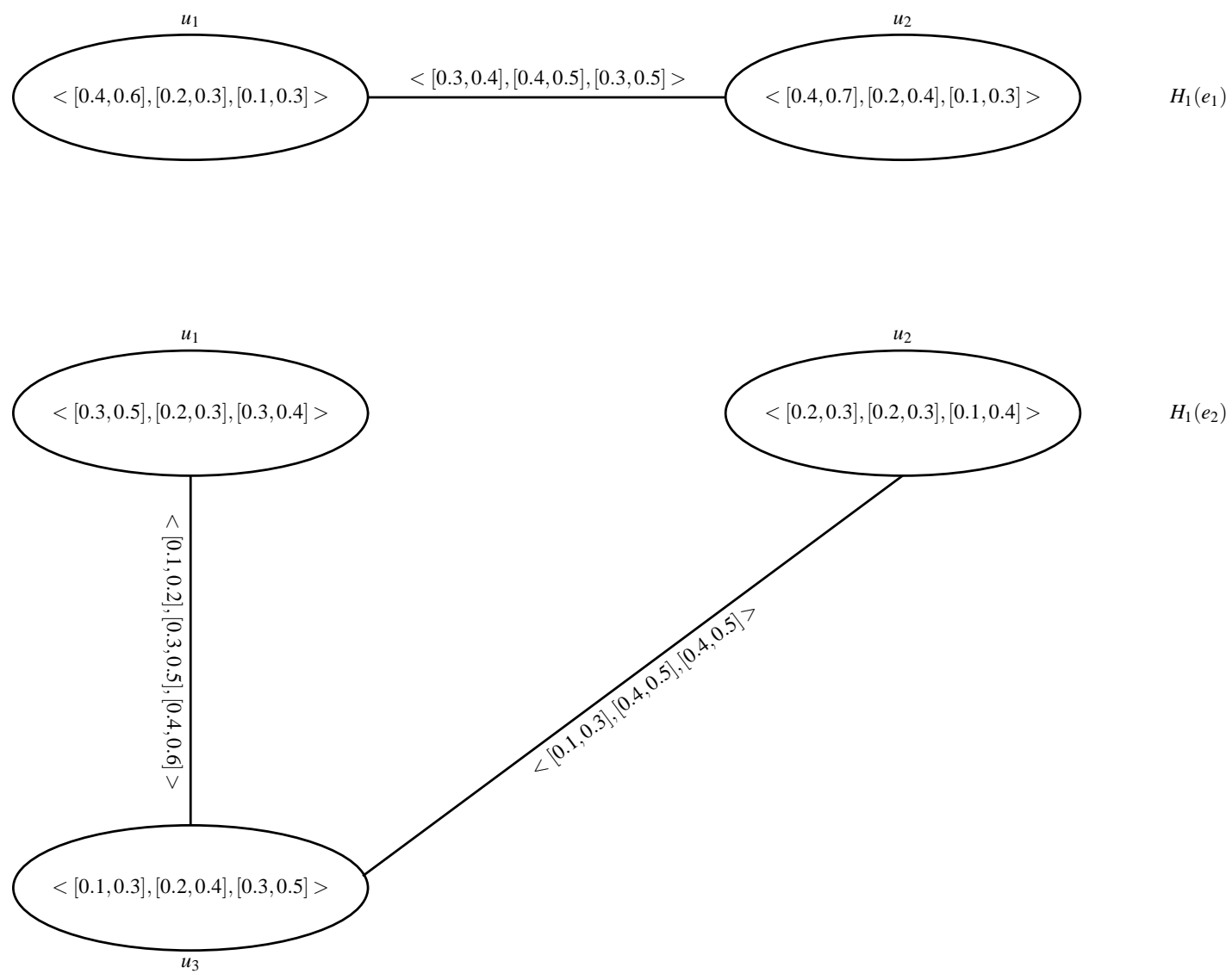

Fig. 5: Interval-valued neutrosophic soft graph $G_{1}$

$H\left(e_{2}\right)=H_{1}\left(e_{2}\right) \cup H_{2}\left(e_{2}\right)$, and $H\left(e_{3}\right)=H_{2}\left(e_{3}\right)$ are interval-valued neutrosophic graphs corresponding to the parameters 

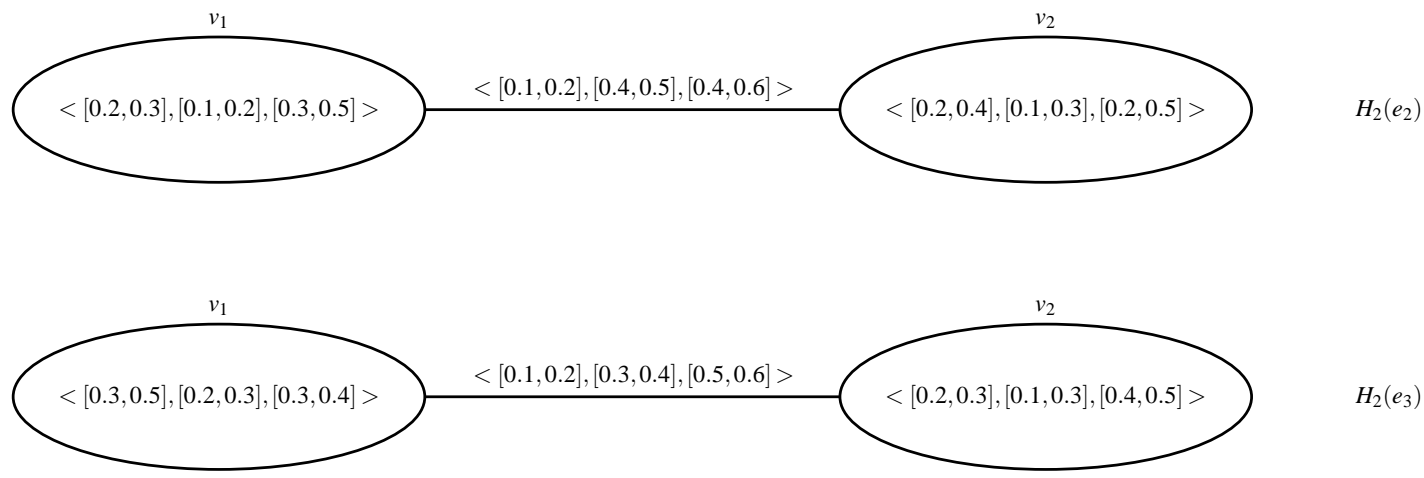

Fig. 6: Interval-valued neutrosophic soft graph $G_{2}$

$e_{1}, e_{2}$ and $e_{3}$. Hence, the union of $G_{1}$ and $G_{2}$ corresponding to the parameter $e_{2}$ is interval-valued neutrosophic soft graph as shown in Figure 7. The figures of $H\left(e_{1}\right)$ and $H\left(e_{2}\right)$ can be drawn similarly.

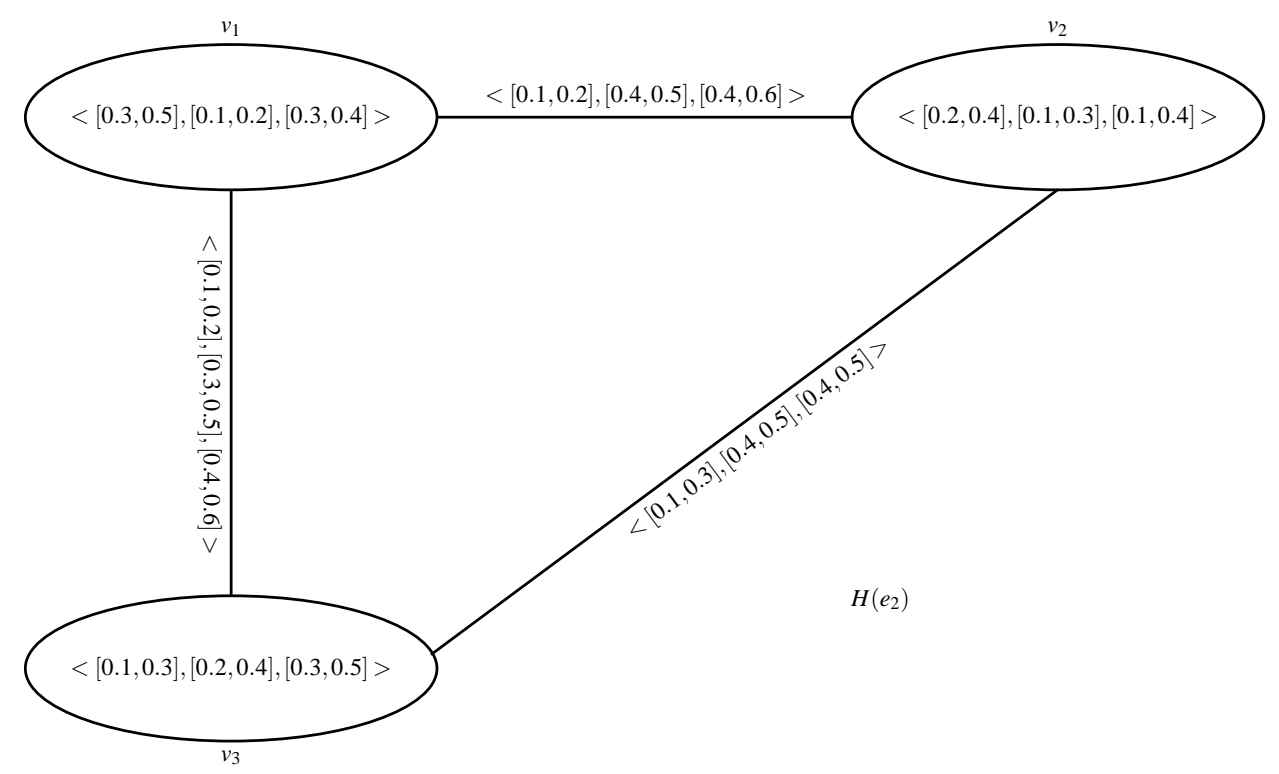

Fig. 7: The union of $G_{1}$ and $G_{2}$ corresponding to the parameter $e_{2}$

Theorem 2. If $G_{1}$ and $G_{2}$ are two interval-valued neutrosophic soft graphs, then so is $G_{1} \cup G_{2}$.

Proof. By using Definition 3.10, it can be shown in a similar way to proof of Theorem 1.

Definition 12. Let $G_{1}=\left(G_{1}^{*}, K_{1}, M_{1}, A\right)$ and $G_{2}=\left(G_{2}^{*}, K_{2}, M_{2}, B\right)$ be two interval-valued neutrosophic soft graphs of simple graphs $G_{1}^{*}=\left(V_{1}, E_{1}\right)$ and $G_{2}^{*}=\left(V_{2}, E_{2}\right)$, respectively. The intersection of $G_{1}$ and $G_{2}$ is denoted by $G_{1} \cap G_{2}=$ 
$\left(G^{*}, K, M, A \cup B\right)$, where $G^{*}=\left(V_{1} \cap V_{2}, E_{1} \cap E_{2}\right)$, and is defined by

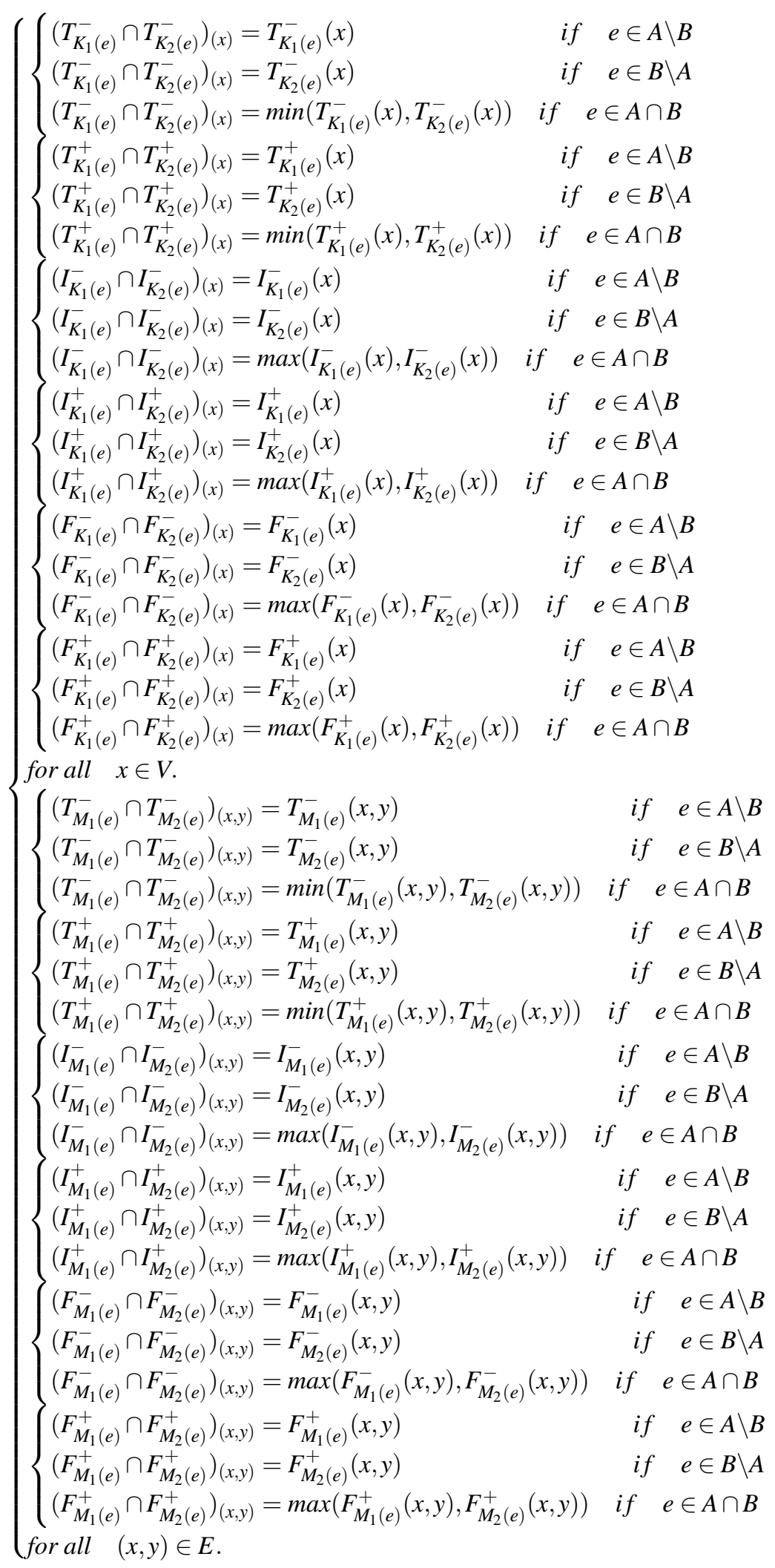


Example 6. Let consider the Example 5. From Definition $11, G_{1} \cap G_{2}=\left(G^{*}, K, M, A \cup B\right)$, where $A \cup B=\left\{e_{1}, e_{2}, e_{3}\right\}$. Also $H\left(e_{1}\right)=H_{1}\left(e_{1}\right), H\left(e_{2}\right)=H_{1}\left(e_{2}\right) \cap H_{2}\left(e_{2}\right)$, and $H\left(e_{3}\right)=H_{2}\left(e_{3}\right)$ are interval-valued neutrosophic graphs corresponding to the parameters $e_{1}, e_{2}$ and $e_{3}$. Hence, the intersection of $G_{1}$ and $G_{2}$ corresponding to the parameter $e_{2}$ is interval-valued neutrosophic soft graph as shown in Figure 8. The figures of $H\left(e_{1}\right)$ and $H\left(e_{2}\right)$ can be drawn similarly.

$$
H\left(e_{2}\right)=H_{1}\left(e_{2}\right) \cap H_{2}\left(e_{2}\right)=\left\{v_{1}\left|([0.2,0.3],[0.2,0.3],[0.3,0.5]), v_{2}\right|([0.2,0.3],[0.2,0.3],[0.2,0.5]\} .\right.
$$
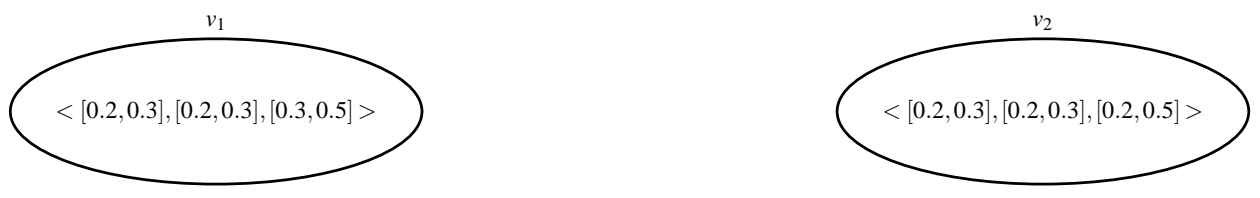

$H\left(e_{2}\right)$

Fig. 8: The intersection of $G_{1}$ and $G_{2}$ corresponding to the parameter $e_{2}$

Theorem 3. If $G_{1}$ and $G_{2}$ are two interval-valued neutrosophic soft graphs, then so is $G_{1} \cap G_{2}$.

Proof. By using Definition 12, it can be shown in a similar way to proof of Theorem 1.

\section{Conclusion}

Graph theory is an extremely useful mathematical tool to solve the complicated problems in different fields. The intervalvalued neutrosophic soft sets constitute a generalization of interval-valued fuzzy soft set theory. The interval-valued neutrosophic soft models give more sensitive, flexibility and conformity to the systems as compared to the interval-valued fuzzy soft models. We applied the concept of interval-valued neutrosophic soft sets to graph structures and describe method of their construction. We also defined cartesian product, union and intersection on interval-valued neutrosophic soft graphs and gave some of their properties. We want to make, in near future, some algorithm and models using these results.

\section{Competing interests}

The authors declare that they have no competing interests.

\section{Authors' contributions}

All authors have contributed to all parts of the article. All authors read and approved the final manuscript.

\section{References}

[1] F. Smarandache, Neutrosophic set-a generalization of the intuitionistic fuzzy set. Granular Computing, 2006 IEEE International Conference, (2006) 38-42.

[2] L. Zadeh, Fuzzy sets. Inform and Control, 8 (1965) 338-353.

[3] K. Atanassov, Intuitionistic fuzzy sets. Fuzzy Sets and Systems, 20 (1986) 87-96.

[4] K. Atanassov and G. Gargov, Interval valued intuitionistic fuzzy sets. Fuzzy Sets and Systems, 31, 343-349 (1989).

[5] H. Wang, F. Smarandache, Y. Zhang and R. Sunderraman, Single valued Neutrosophic Sets. Multispace and Multistructure, 4 (2010) 410-413. 
[6] H .Wang, Y. Zhang and R. Sunderraman, Truth-value based interval neutrosophic sets. Granular Computing, 2005 IEEE International Conference, 1 (2005) 274-277.

[7] A. Q. Ansari, R. Biswas and S. Aggarwal, Neutrosophic classifier: An extension of fuzzy classifier. Applied Soft Computing, 13 (2013) 563-573.

[8] H. Zhang, J. Wang and X. Chen, An outranking approach for multi-criteria decision-making problems with interval-valued neutrosophic sets. Neural Computing and Applications, 27(3) (2015) 1-13.

[9] H.Y. Zhang, P. Ji, J. Q. Wang and X. HChen, An Improved Weighted Correlation Coefficient Based on Integrated Weight for Interval Neutrosophic Sets and its Application in Multi-criteria Decisionmaking Problems. International Journal of Computational Intelligence Systems, 8(6) (2015).

[10] I. Deli, M. Ali and F. Smarandache, Bipolar neutrosophic sets and their application based on multicriteria decision making problems, Advanced Mechatronic Systems (ICAMechS), International Conference, (2015) 249-254.

[11] M. Ali and F. Smarandache, Complex Nuetrosophic Set. Neural Computing and Applications, 27(1) (2016) 1-18.

[12] M. Ali, I. Deli and F. Smarandache, The Theory of Neutrosophic Cubic Sets and Their Applications in Pattern Recognition. Journal of Intelligent and Fuzzy Systems, 30(4) 2016 1957-1963.

[13] D. Molodtsov, Soft set theory-first results, Computers and Mathematics with Applications, 37 (1999) 19-31.

[14] P.K Maji, R. Biswas and A.R. Roy, Fuzzy Soft Set, The Journal of Fuzzy Mathematics, 9(3) (2001) 677-692.

[15] X. Yang, T. Y. Lin, J. Yang, Y. Li and D. Yu, Combination of interval-valued fuzzy set and soft set. Computers and Mathematics with Applications, 58(3) (2009) 521-527.

[16] P.K. Maji, Neutrosophic soft set. Annals of Fuzzy Mathematics and Informatics, 5(1) (2013) 157-168.

[17] I. Deli, Interval-valued neutrosophic soft sets and its decision making, International Journal of Machine Learning and Cybernetics, 8 (2015) 1-12.

[18] L. Euler, Solutio problematis ad geometriam situs pertinentis, Commentarii Academiae Scientiarum Imperialis Petropolitanae, 8 (1736) 128-140.

[19] S.Broumi, M. Talea, A.Bakali and F. Smarandache, Interval Valued Neutrosophic Graphs, Critical review, 12 (2016) 5-33. 